\title{
Comparison of Active Drag Using the MRT-Method and the MAD-System in Front Crawl Swimming ${ }^{+}$
}

\author{
Kenzo Narita ${ }^{1, *}$, Futoshi Ogita ${ }^{2}$, Motomu Nakashima ${ }^{3}$ and Hideki Takagi ${ }^{4}$ \\ 1 Doctoral Program in Physical Education, Health and Sport Sciences, University of Tsukuba, \\ Tsukuba 305-8574, Japan \\ 2 Department of Sports and Life Sciences, National Institute of Fitness and Sports in Kanoya, \\ Kanoya 891-2393, Japan; ogita@nifs-k.ac.jp \\ 3 Department of Systems and Control Engineering, Tokyo Institute of Technology, Tokyo 152-8550, Japan; \\ motomu@sc.e.titech.ac.jp \\ 4 Faculty of Health and Sport Sciences, University of Tsukuba, Tsukuba 305-8574, Japan; \\ takagi.hideki.ga@u.tsukuba.ac.jp \\ * Correspondence: ninzen7@gmail.com; Tel.: +81-80-4337-4699 \\ + Presented at the 12th Conference of the International Sports Engineering Association, Brisbane, \\ Queensland, Australia, 26-29 March 2018.
}

Published: 11 February 2018

\begin{abstract}
The purpose of this study was to compare the active drag values estimated by the MRT-method and the MAD-system. Six male competitive swimmers participated in this study and performed front crawl with arms only condition. The drag was compared at six-staged velocities ranged from 0.9 to $1.4 \mathrm{~m} / \mathrm{s}$ between MRT-method and MAD-system. The drag estimated by MRT-method showed larger values than that obtained using MAD-system at each velocity (MRT-method/MAD-system: $119 \%$ at $1.0 \mathrm{~m} / \mathrm{s} ; 133 \%$ at $1.2 \mathrm{~m} / \mathrm{s} ; 147 \%$ at $1.4 \mathrm{~m} / \mathrm{s}$ ). In addition, the stroke length in MRT-method condition decreased with swimming velocity being increased, while that in MAD-system condition was constant. Therefore, swimmers had to increase their stroke frequency in MRT-method condition in order to achieve the same swimming velocities as MADsystem condition, especially at high velocities. It was concluded that the difference in the way of exerting propulsion between MAD-system and MRT-method influenced the active drag which were estimated in two methods.
\end{abstract}

Keywords: active drag; front crawl; stroke frequency; stroke length; propulsion

\section{Introduction}

A resistive force in swimming, i.e., active drag, is a main factor of determining the swimming performance. Swimming velocity depends on a balance between propulsive force and active drag. When active drag being larger than propulsive force in swimming, the velocity of the swimmer decreases. Hence, reducing active drag is important to achieve high swimming velocity. Although various methodologies have been suggested, it is currently impossible to measure active drag accurately during swimming, and there has been no standard method for measuring active drag.

In front-crawl swimming, which is the fastest human swimming stroke, distances varied from $50 \mathrm{~m}$ to $1500 \mathrm{~m}$ are adopted as official events in competitions. Since swimmers alter their swimming velocity depending on the distance, it is necessary to evaluate active drag at various swimming velocities to provide detailed kinetic information on swimming performances to athletes and coaches. As a methodology for evaluating active drag at various velocities, Hollander et al. [1] developed the measuring active drag system (MAD-system). In this method, the swimmer propelled him/herself forward by pushing the pads which were fixed under the water, and the pushing forces exerted by the swimmer was measured by a load cell. Then, under the assumption that the swimmer's 
swimming velocity during the measurement is constant, the active drag was evaluated from the principle that the mean propulsive force exerted by the swimmer (that is, the force for pushing the pad) was equal to the mean drag in swimming. The drag estimation using MAD-system can only be applied to front crawl swimming without legs motion due to its methodological characteristics. Therefore, active drag acting on the swimmer during whole body swimming cannot be estimated with this method. Another problem in MAD-system is that swimmers change their velocity in a different manner compared with actual swimming condition. Swimming velocity is calculated by the product of stroke frequency and length. In MAD-system condition, however, stroke length of the swimmer is constant and the swimming velocity is affected only by the stroke frequency, which is not the case in reality [2,3]. On the other hand, the drag in various swimming styles can be assessed by other two methods $[4,5]$. However, they can only evaluate the drag during maximal effort trials. The drag during swimming has been mainly assessed by those three methods, and MAD-system is the only method which is able to evaluate active drag at various velocities among the three.

A methodology for estimating the drag in swimming using measured values of residual thrust (MRT-method) has recently been developed by Narita et al. [6]. The MRT-method has no restriction on swimming style and velocity, therefore, the method can evaluate active drag at any velocities as with MAD-system. Furthermore, unlike the MAD-system approach, this method allows researchers to verify an influence of stroke frequency and length on active drag. In MRT-method condition, the swimmer propels his/her body forward by sweeping their arms through the water without the restriction by the pads. Therefore, with this method, it is possible to investigate the active drag at various velocities without neglecting the influence of the stroke length. By comparing active drag evaluated by both MAD-system and MRT-method with the same swimmers, the effect of the way of generating propulsion on the active drag can be investigated.

The purpose of this study was to compare the active drag values in front crawl swimming with arms only condition between the MRT-method and the MAD-system.

\section{Methods}

\subsection{Participants}

Six male competitive swimmers (age: $20.0 \pm 1.0$ years; height: $1.71 \pm 0.03 \mathrm{~m}$; weight: $67.6 \pm 6.2 \mathrm{~kg}$ ) participated in this study. They all trained six days a week and had experience in participating in Japanese national competitions. The test procedures were approved by the University of Tsukuba Ethics Committee and each participant signed an informed-consent form.

\subsection{Experimental Design}

Each swimmer performed front crawl using arms only in MRT-method and MAD-system. To restrict the movement of the swimmer's legs, we attached a buoyant buoy with the thigh of the swimmer and fastened a band to the ankle. In all experiments, the swimmers used a snorkel to eliminate an influence of the breathing motion. Moreover, the swimmers were instructed to wear the same type of swimsuit in both testing conditions to avoid any potential effects on resistance caused by different types of swimsuit.

\subsubsection{MRT-Method}

Trials using MRT-method were conducted in a water flume (Igarashi Industrial Works Co., Ltd., Chiba, Japan), which allowed the flow velocity to be controlled precisely. Prior to the measurements, the swimmers had familiarization period for the flume.

The MRT-method evaluates the active drag from the relationship between the residual thrusts and the flow velocities. The residual thrust is the difference between the propulsion and drag. This can be calculated by measuring towing forces exerted by the swimmer by two load cells connected to wires which are attached to the waist of the swimmer at various flow velocities $(U)$, while the swimmer maintains the same techniques and kinematics. Thus, to estimate the active drag at a given velocity $=i \mathrm{~m} / \mathrm{s}\left(V_{s i}\right)$, the swimmer has to maintain his/her stroke motion and body position required 
to swim at $V_{\mathrm{si}}$ even when $U$ was varied. Prior to measuring the residual thrust, each swimmer self-propelled in the flume with the flow velocity $U$ being set to $i \mathrm{~m} / \mathrm{s}$. To make it easy for the swimmer to maintain his/her stroke at different $U$, the stroke time (s/stroke) that the swimmer used to propel himself at $i \mathrm{~m} / \mathrm{s}$ was provided using a small audible waterproof metronome (Tempo trainer Pro; FINIS, Inc., Livermore, LA, USA). To measure the residual thrust at each $U$, a belt wrapped around the swimmer's waist was connected to load cells using wires (LUX-B-2KN-ID, Kyowa Electronic Instruments Co. Ltd., Tokyo, Japan). The load cells were located at the front and back of the flume (Figure 1). The forward and backward towing forces were measured for $10 \mathrm{~s}$, and then the residual thrust was calculated from their difference between the forces. We measured the residual thrust at eight points within the range of $\pm 0.2 \mathrm{~m} / \mathrm{s}$ around $V_{s i}$, changing $U$ by $0.05 \mathrm{~m} / \mathrm{s}$ each time. Thereafter, we derived best-fit regression curves for the measured values of residual thrust and $U$ and used them to calculate the active drag (for further details, see Narita et al. [6]). The drag was estimated in six-staged velocities from 0.9 to $1.4 \mathrm{~m} / \mathrm{s}$. The stroke frequency (SF [Hz]) was calculated from the inverse of the stroke time (s/stroke), and the stroke length SL was computed by dividing the velocities $U_{\text {Treo }}$ derived from the regression curve fitted to the $S F$.

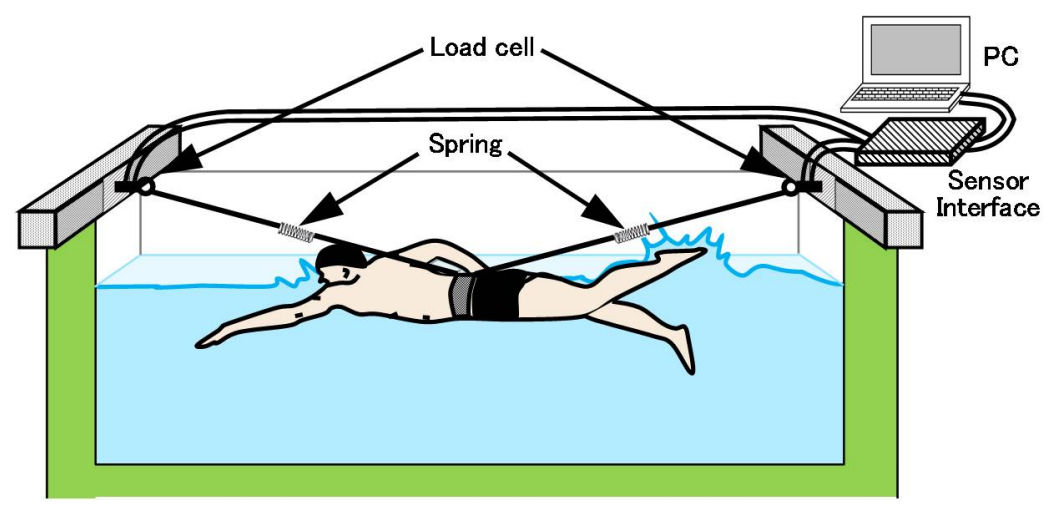

Figure 1. Top view of MRT-method.

\subsubsection{MAD-System}

In testing using MAD-system, each swimmer swam $25 \mathrm{~m}$ with pushing pads. The pads were attached to a $23 \mathrm{~m}$ rod, which was mounted $0.8 \mathrm{~m}$ below the water surface and connected to a force transducer, and a $1.30 \mathrm{~m}$ interval (Figure 2). The force by pushing off pads were measured at a frequency of $100 \mathrm{~Hz}$. Mean active drag was determined to be equal to the mean propulsive force under the assumption that the velocity of the swimmer was constant throughout the trial. To establish the relationship between the active drag and the velocity, each subject completed ten trials at different selected velocities with approximately $3 \mathrm{~min}$ rest between trials. We chose six out of the ten trials which showed similar velocity as $U_{\text {Treo }}$ that was calculated for the same swimmer in MRT-method. The SF was calculated by dividing the swimming velocity by the SL (which is constantly $2.6 \mathrm{~m}$ with MAD-system).

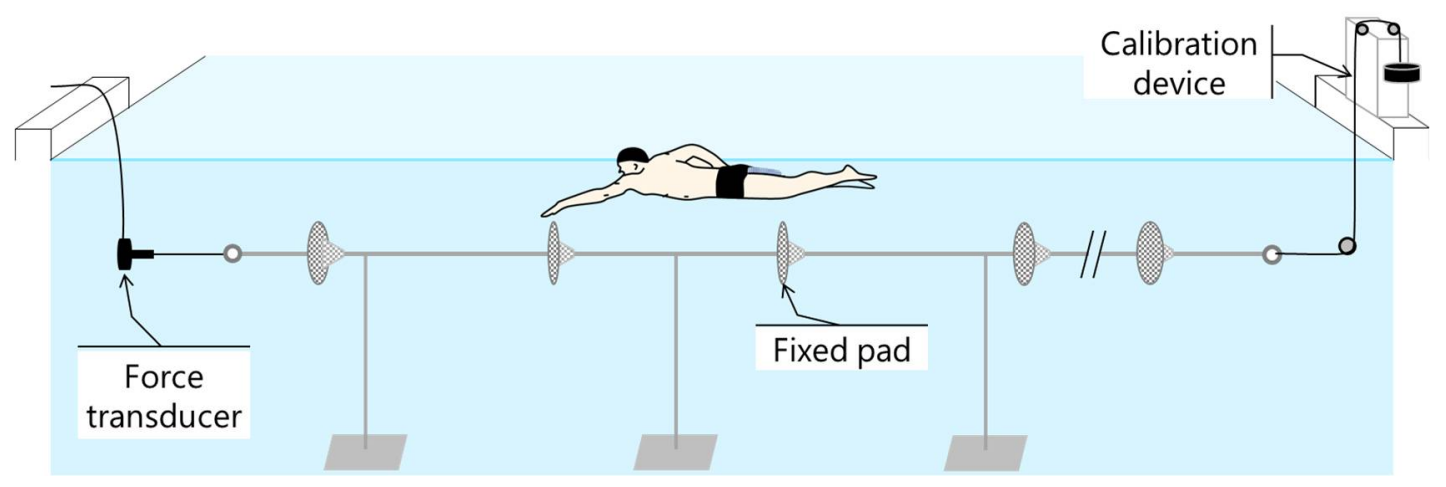

Figure 2. Top view of MAD-system. 


\subsection{Data Analysis}

To compare the values of active drag which were evaluated with MRT-method and MAD-system at various velocities, the drag/velocity data were fitted to the function: $D=k v^{n}$ ( $D:$ drag, $v$ : velocity) to obtain coefficient $k$ and degree $n$ for each swimmer, and the active drag for 1.0, 1.2 and $1.4 \mathrm{~m} / \mathrm{s}$ were calculated by the aforementioned equation with obtained $k$ and $n$ values being substituted.

\subsection{Statistical Analysis}

We compared the active drag calculated by MRT-method and MAD-system using a paired $t$ test. To investigate the influence of stroke parameters on the swimming velocity and the active drag in MRT-method and MAD-system, we obtained by Pearson's correlation coefficients $(r)$ between swimming velocity/active drag and SF/SL of all swimmers. All statistical analyses were conducted at a significance level of $p<0.05$ using SPSS ver. 22.0 (SPSS, Inc., Chicago, IL, USA).

\section{Results}

Table 1 indicates the values of $k$ and $n$ obtained in MRT-method and MAD-system. Significant differences between the two methods were observed in $k(t(5)=4.96, p<0.01)$ and $n(t(5)=2.76$, $p=0.04)$. The average values of all swimmer's active drag at 1.0, 1.2 and $1.4 \mathrm{~m} / \mathrm{s}$ were shown in Figure 3. There were also significant differences in active drag values at $1.0(t(5)=4.96, p<0.01), 1.2$ $(t(5)=5.00, p<0.01)$ and $1.4 \mathrm{~m} / \mathrm{s}(t(5)=3.90, p=0.01)$.

Table 1. Coefficient $k$ and degree $n$ in each swimmer obtained by MRT-method and MAD-system.

\begin{tabular}{ccccc}
\hline \multirow{2}{*}{ Swimmer } & \multicolumn{2}{c}{ MRT-Method } & \multicolumn{2}{c}{ MAD-System } \\
\cline { 2 - 5 } & $\boldsymbol{k}$ & $\boldsymbol{n}$ & $\boldsymbol{k}$ & $\boldsymbol{n}$ \\
\hline A & 37.8 & 3.28 & 30.5 & 1.73 \\
B & 34.1 & 2.30 & 28.2 & 1.85 \\
C & 32.2 & 2.34 & 27.1 & 2.06 \\
D & 38.9 & 2.16 & 29.6 & 2.10 \\
E & 33.0 & 2.83 & 31.3 & 1.85 \\
F & 31.1 & 2.23 & 27.6 & 1.84 \\
\hline Mean & 34.5 & 2.53 & 29.1 & 1.90 \\
SD & 2.9 & 0.40 & 1.5 & 0.13 \\
\hline
\end{tabular}

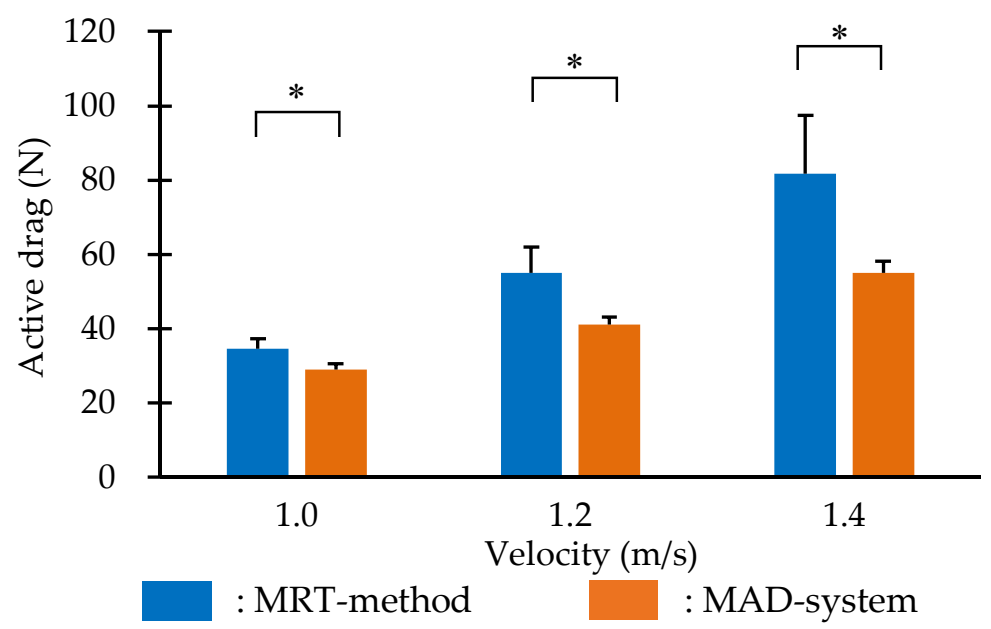

Figure 3. The average values of active drag at 1.0, 1.2 and $1.4 \mathrm{~m} / \mathrm{s}$ in MRT-method (blue) and MADsystem (orange). Asterisk $\left(^{*}\right)$ indicates a significant difference between MRT-method and MADsystem, $p<0.05$. 
Figure 4 present the relationships between swimming velocity/active drag and stroke frequency. In MRT-method, SF had a significant positive correlation with swimming velocity $(r=0.797, p<0.01)$ and active drag $(r=0.808, p<0.01)$, whereas SL showed a significant negative correlation with swimming velocity $(r=-0.401, p=0.02)$ and active drag $(r=-0.452, p<0.01)$. On the other hand, in MAD-system, there was a significant positive correlation between SF and swimming velocity $(r=$ 0.999, $p<0.01) /$ active drag $(r=0.970, p<0.01)$, besides, the relationship between SL and active $\mathrm{drag} /$ velocity could not be evaluated because SL was constant.

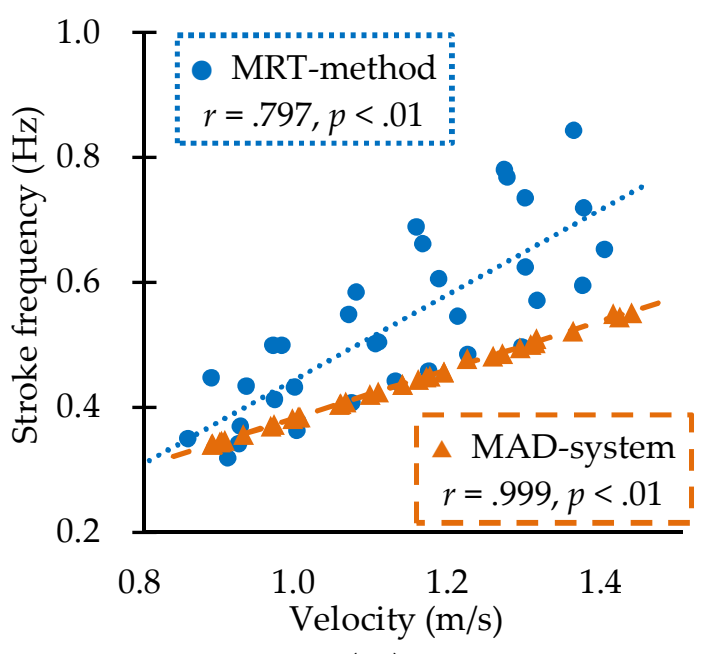

( a )

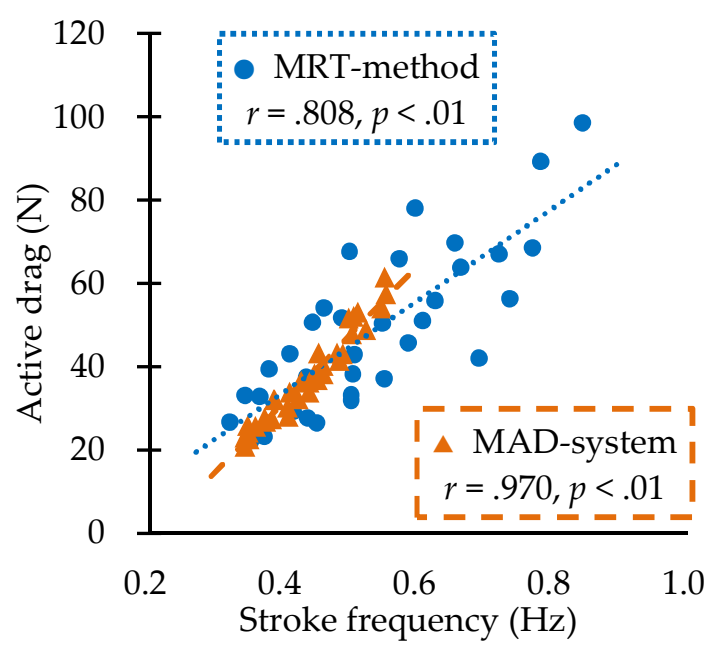

(b)

Figure. 4 The relationship between each variables in MRT-method (blue) and MAD-system (orange) for all swimmers. (a) The relationship between swimming velocity and stroke frequency; (b) the relationship between stroke frequency and active drag.

\section{Discussion}

Active drag in front-crawl swimming without legs motion assessed by MRT-method was larger than the drag estimated using MAD-system at all velocities. In MAD-system, due to its mechanical structure, swimmers propels their body by pushing fixed pads under the water. Thus, swimmers can utilize all reaction force acquired by pushing the fixed pads by the hands as the propulsive force. On the other hand, during the actual swimming, the force swimmers obtain from the water are divided into propulsive force and force which does not contribute to propulsion [7]. Therefore, propulsive force is probably generated more efficiently by swimmers with MAD-system compared with the actual swimming and MRT-method condition at the same velocities. The swimming velocity is determined by the balance between propulsive force and active drag. It means that when the swimmer achieves a given swimming velocity with a small propulsive force, the drag acting on the swimmer is also small. In this study, the SL, which was adopted as a simple index of swimming efficiency, was constant regardless the swimming velocity in MAD-system condition. However, in MRT-method, it decreased with the velocity being increased. Therefore, MRT-method required the swimmers to achieve the same swimming velocities as they did with MAD-system by increasing their $\mathrm{SF}$, especially at high velocity conditions. On the other hand, SF and active drag were positively correlated in both methods. However, the resistance force is strongly influenced by the velocity, as well as SF. Hence, in order to investigate a relationship between SF and the drag with minimizing the influence of the velocity, we calculated an active drag coefficient and conducted a correlation analysis. A significant positive correlation was observed between these variables in MRT-method $(r=0.44, p<0.01)$, while no significant correlation was found in MAD-system $(r=-0.10, p=0.57)$. In front-crawl swimming, swimmers repeatedly move their arms and legs around the water surface. Therefore, it is expected that additional drags from waves and splashes are generated in each stroke cycle. Therefore, it is possible that the active drag in high SF conditions was affected by those drags more than that in low SF conditions. 
On the other hand, since present study did not conduct a motion analysis, the influence of the difference in the motion of the swimmer between each method on the active drag was unclear. Obtaining information on the path and speed of upper limbs and pitch/yaw of the body of swimmers in each method will be helpful for better understanding of the influence of these variables on the difference of the drag between the two methods. Therefore, further investigations including the motion analysis are needed for detailed analysis of the effect of the difference of each methodology on active drag.

\section{Conclusions}

The present study compared the active drag using the MRT-method and the MAD-system in front crawl swimming without kicking motion. As a result, the active drag values estimated using MRT-method was higher than those obtained by MAD-system. In addition, SL in MAD-system condition was constant, while that in MRT-method condition decreased with swimming velocity being increased. Therefore, swimmers had to increase their SF in MRT-method condition in order to achieve the same swimming velocities as MAD-system condition, especially in high velocity conditions. It is probable that the different ways to generate propulsive force by upper limbs between the two methods influence the stroke parameters, consequently, the active drag.

Acknowledgments: This research was supported in part by the Ministry of Education, Culture, Sports, Science and Technology (MEXT) for the Human High Performance Project (2014-2018) and by a grant from Advanced Research Initiative for Human High Performance (ARIHHP), University of Tsukuba.

Conflicts of Interest: There are no conflicts of interest to declare.

\section{References}

1. Hollander, A.P.; De Groot, G.; van Ingen Schenau, G.; Toussaint, H.M.; De Best, H.; Peeters, W.; Meulemans, A.; Schreurs, A.W. Measurement of active drag during crawl arm stroke swimming. J. Sports Sci. 1986, 4, 21-30.

2. Craig, A.B.; Skehan, P.L.; Pawelczyk, J.A.; Boomer, W.L. Velocity, stroke rate, and distance per stroke during elite swimming competition. Med. Sci. Sports Exerc. 1985, 17, 625-634.

3. Seifert, L.; Toussaint, H.M.; Alberty, M.; Schnitzler, C.; Chollet, D. Arm coordination, power, and swim efficiency in national and regional front crawl swimmers. Hum. Mov. Sci. 2010, 29, 426-439.

4. Kolmogorov, S.; Duplishcheva, O. Active drag, useful mechanical power output and hydrodynamic force coefficient in different swimming strokes at maximal velocity. J. Biomech. 1992, 25, 311-318.

5. Formosa, D.P.; Mason, B.; Burkett, B. The force-time profile of elite front crawl swimmers. J. Sports Sci. 2011, 29, 811-819.

6. Narita, K.; Nakashima, M.; Takagi, H. Developing a methodology for estimating the drag in front-crawl swimming at various velocities. J. Biomech. 2017, 54, 123-128.

7. Toussaint, H.M. Differences in propelling efficiency between competitive and triathlon swimmers. Med. Sci. Sports Exerc. 1990, 22, 409-415.

(C) 2018 by the authors. Licensee MDPI, Basel, Switzerland. This article is an open access article distributed under the terms and conditions of the Creative Commons Attribution (CC BY) license (http://creativecommons.org/licenses/by/4.0/). 